\title{
Combined High and Low-Thrust Geostationary Orbit Insertion with Radiation Constraint
}

\author{
Malcolm Macdonald ${ }^{1}$ and Steven Robert Owens ${ }^{2}$ \\ University of Strathclyde, Glasgow G1 1XJ, Scotland
}

\begin{abstract}
The sequential use of an electric propulsion system is considered in combination with a high-thrust propulsion system for application to the propellant-optimal Geostationary Orbit insertion problem, whilst considering both temporal and radiation flux constraints. Such usage is found to offer a combined propellant mass saving when compared with an equivalent high-thrust only transfer. This propellant mass saving is seen to increase as the allowable transfer duration is increased, and as the thrust from the low-thrust system is increased, assuming constant specific impulse. It was found that the required plane change maneuver is most propellant-efficiently performed by the high-thrust system. The propellant optimal trajectory incurs a significantly increased electron flux when compared to an equivalent high-thrust only transfer. However, the electron flux can be reduced to a similar order of magnitude by increasing the high-thrust propellant consumption, whilst still delivering an improved mass fraction.
\end{abstract}

Keywords: Orbit Insertion, Radiation Constraint, Mission analysis, System analysis, Hybrid propulsion.

\section{Introduction}

The use of low-thrust electric propulsion (EP) on-board telecommunications spacecraft in Geostationary orbit (GEO) for station-keeping is well-established. Similarly, GEO orbit insertion using only low-thrust electric propulsion has been extensively studied [1-13]. In the frame of Horizon 2020 project HYPROGEO, which aims to develop synergies between electrical and hybrid propulsion (solid polyethylene fuel with high concentration liquid $\mathrm{H}_{2} \mathrm{O}_{2}$ oxidizer) systems, in this paper the use of the EP systems is considered in combination with a high-thrust propulsion system for application to the propellant-optimal GEO insertion problem, whilst considering both temporal and radiation flux constraints.

There has been prior research focused on coupling high and low-thrust propulsion technologies to form a hybrid propulsion system; note that in this context, and for the remainder of this paper, hybrid is used to mean combination of different thrust and specific impulse levels. Specifically in this paper, high and low thrust combined with low and high specific impulse, respectively. The advantages of coupling are obvious; reducing the transfer time compared to only low-thrust propulsion, providing a propellant mass saving compared to only chemical propulsion

\footnotetext{
${ }^{1}$ Reader of Space Technology, Strathclyde Space Institute, Department of Mechanical \& Aerospace Engineering, University of Strathclyde, James Weir Building, 75 Montrose Street, Glasgow G1 1XJ, Scotland.

Corresponding author: malcolm.macdonald.102@strath.ac.uk

${ }^{2}$ Knowledge Exchange Fellow, Strathclyde Space Institute, Department of Mechanical \& Aerospace Engineering, University of Strathclyde, James Weir Building, 75 Montrose Street, Glasgow G1 1XJ, Scotland.
} 
and reducing the time the spacecraft spends in the Van Allen radiation belts, which can cause significant power degradation to the solar panels.

The first indication of using high and low-thrust systems together was in 1962 by Theodore Edelbaum [14], around the time when EP systems were starting to be considered as a feasible propulsion system for spacecraft [15]. Early work on the transfer method, such as the analysis in [14], assumed the high-thrust segments were impulsive and patched with the low-thrust transfers to form the trajectory. This was also the case in proceeding work [16,17]. In using this method, the transversality condition used in the optimization process offered some conditions for patching the segments together. Other work has made use of primer vector theory, which can provide similar results; however in a more general and direct manner without the need to patch sections together [18]. This was based on a switching function that could be used to switch between propulsion systems and also allow the spacecraft to enter a coast period. This switching methodology was also identified in [19] and [20] and the similarities between [18-20] were described in [21].

Work has also considered the practicalities of implementing hybrid propulsion systems, that is, performing an analysis based on launch vehicle technology, spacecraft power availability and efficiencies of current low thrust systems. This has allowed for realistic studies of transfers from Earth to the Moon using chemical-electric systems and also nuclear-electric systems [22-24]. These studies have again identified the advantage of such a propulsion concept. In addition to Earth - Moon transfers, there have also been studies of combined propulsion systems for orbit transfers within the Earth's sphere of influence; most notably, transfers to GEO. This research has considered the use of a chemical system on-board the satellite in conjunction with an EP system, also on-board the satellite but for the main use of station keeping maneuvers, to perform part of the orbit raise maneuver [25-27]. In addition, work has considered the effect of the launch vehicle on the transfer [28]. In order to maximize the satellite dry mass and hence minimize the propellant mass, these studies have also considered the effect of varying the propulsion system specification which has shown there could be some advantage to an EP system with variable specific impulse and thrust. The work in [28] also found the optimum EP system specific impulse depends strongly on the chemical propulsion system specific impulse which is due to a trade-off between minimum propellant mass and minimum time. This is interesting as the hybrid propulsion analysis herein is dependent on the ratio of the high and low-thrust system's specific impulses; however, a similar trend is not observed as the analysis considers a minimum propellant optimization satisfying a user specified time constraint.

Power system degradation due to radiation was studied in [29] where it was confirmed the time spent in the radiation belts can be reduced drastically by employing a hybrid system as opposed to low-thrust only. The introduction of the high-thrust system does impose a mass penalty but this can be offset again by the reduction in radiation effects on the spacecraft.

Prior studies of hybrid GEO insertion scenarios have mainly focused on transfers that use high-thrust burns to achieve an intermediate orbit someway between the initial injection and target orbits. This method then uses an outward spiral trajectory towards the target orbit. However, by considering the bi-elliptic transfer ethos, see [30-33], and how it can save propellant mass by using an intermediate orbit far greater than the target orbit, a similar hybrid transfer is also considered herein. In this case, the high-thrust section is used to propel the spacecraft beyond the target to an intermediate orbit, with both perigee and apogee larger than the target, and then the low-thrust propulsion system is used to spiral back in-towards the target orbit. Such a transfer is called a Hohmann Spiral Transfer, HST [34-38], and is expected to yield significant benefits when the radiation flux is incorporated as a constraint to the trajectory design. Furthermore, as the space debris mitigation guidelines state that no object should be jettisoned into an orbit that intersects the GEO ring $[39,40]$, it means that such an intermediate orbit beyond GEO may be used to jettison the exhausted high-thrust propulsion system, hence further increasing the efficiency of the low-thrust system by reducing the spacecraft mass.

It should be noted that throughout this analysis, and much of the literature, it is assumed that the high-thrust burns are executed fully before the low-thrust phase begins, separating the trajectory into two clearly phases. Whilst it is likely that the propellant optimal solution would intertwine these two phases, the operation of the low-thrust 
system is highly likely to require deployed solar array panels, which would in-turn be incompatible with the operation of a high-thrust propulsion system. Thus, such a propellant optimal solution is highly likely to be a suboptimal system-wide solution.

\section{Hybrid Trajectory Optimization}

Several of the papers previously discussed considered different methods of addressing the issues associated with optimization of hybrid low and high-thrust propulsion transfers. For example, there has been research that used a pre-calculated transfer array that can be interpolated in order to speed up the transfer analysis [26,27]. The initial analysis in this method was therefore computationally heavy but for any other transfers it meant there was a rapid estimation available. This method obviously has a speed advantage but it is limited as it can only evaluate transfers in the region of the initial analysis. The accuracy of the resultant interpolated solutions is also dependent on the discretization of the initial solutions. Other papers have used a patching method allowing the high and low-thrust transfer sections to be decoupled, reducing the overall optimization complexity as each section is analyzed individually as discussed previously [22-24]. Although this patching method can offer near-optimal solutions, it still requires a large amount of user time and knowledge to ensure the different trajectories can be connected. However, a program has been developed to optimize a full hybrid propulsion transfer. This is called SEPSPOT and was developed at the NASA Glen research facility [41]. In coupling the high thrust section this program assumes the spacecraft begins in a circular orbit and can impart a maximum of two high-thrust impulses before the low-thrust system is activated. The total velocity change for the high-thrust section is specified and if the first required impulse is equal or greater than this then the high-thrust section is restricted to one impulse. If the first required impulse is less, then the transfer is a two-burn orbit raise. In this case, the second burn is the difference between the total specified velocity change and the first burn velocity change. Several problems have been identified with SEPSPOT however, specifically related to its sensitivity to the initial guesses and convergence problems [42].

To avoid the need for user input and sensitivity issues, this study employees a hybrid propulsion transfer optimizer that models the high-thrust phase as a Hohmann transfer and generates the low-thrust trajectory using locally optimal control laws.

\subsection{Low-Thrust Trajectory Design}

Where high-thrust transfers are relatively straightforward to predict due to their impulsive nature, low-thrust transfers require continuous thrust to generate a similar velocity requirement, which can result in many orbital revolutions. This spiral trajectory leads to numerically intensive methods in order to determine a solution to any particular transfer problem. Several techniques have therefore been developed which reduce their complexity and produce trajectories that are near optimal. These methods are good for determining an initial solution to a proposed trajectory problem or forming an initial guess to be used as part of a detailed optimization study.

Locally optimal control laws have been used for trajectory generation herein. These control laws maximize the rate of change of a given orbit element and can be specified in closed analytical form as they can be developed from the variational equations of the orbital elements. This can then be used as the optimal thrust direction vector. The advantage of such control laws is the speed of which they can be implemented in a trajectory problem, whilst the primary disadvantage is the sub-optimal nature of the resulting solution. Previous work developed a form of the control laws for semi-major axis, inclination and radius of perigee using the equinoctial elements to avoid singularities associated with the classical elements [6]. In [42], this was extended to include the eccentricity control law which, combined with orbital averaging, was used in an optimization process. The control laws were then explained in an analytical form by the same author in [43]. The control laws have also been derived for use with another form of low-thrust propulsion: solar-sailing [44]. This work was further extended to define the control laws in modified equinoctial elements [45-47] and applied them to many novel missions only possible with a solar-sail [48-52]. 
Due to the quick implementation and versatility of locally optimal control laws, they will be used within this technical note whenever trajectory design is required. Although they are sub-optimal by nature, it was demonstrated in $[6,42,51]$ they exhibit an accuracy $\leq 2.5 \%$ from the optimal solution.

\subsection{Numerical Analysis and Optimization}

The equations of motion are defined in modified equinoctial elements, which are derived in [53] and validated in $[54,55]$. These are used to propagate the trajectory as they are non-singular except for rectilinear orbits when the inclination, $i=\pi$ radians and provide runtime improvements over classical elements for certain orbit transfers [53]. The numerical method propagates the spacecraft position in time using an explicit variable step size Runge-Kutta $(4,5)$ formula known as the Dormand-Prince pair [56]. It is a one-step solver, meaning it only requires the solution at the immediately preceding time point to solve the current point. A relative and absolute tolerance of $10^{-5}$ was chosen for all trajectory propagation and optimization studies to allow rapid analysis without loss of accuracy. It should be noted that a sensitivity analysis was performed to support prior work [34-38] and the relative and absolute tolerance used are a function of that sensitivity analysis. It is also worth noting that the code used was written to ensure similar dimensions across the equations of motion to again address the need for smaller tolerances. No orbit perturbations other than the spacecraft thrust are considered.

The trajectory optimization process is primarily aimed at optimizing the complex low-thrust section of the trajectory; however, it can be modified to include an optimization of the high-thrust section so that the trajectory can be optimized as a full hybrid transfer. This allows the optimizer to choose the 'best' scenario without the need for experienced engineering judgment when splitting the high and low-thrust phases. The optimization algorithm selected uses a constrained nonlinear optimization technique adapting a sequential quadratic programming (SQP) method. This is selected as it has a strict feasibility with respect to the bounds meaning every iterative step is taken within the specified limits [57]. This is necessary for this study as the parameters to be optimized cannot be negative otherwise the trajectory generation will fail. The optimization problem is specified as

$$
\min _{W_{G i} R 2_{i} \theta}\left\{m_{H S T F}\right\}
$$

where $W_{\sigma}$ represents each of the locally optimal control law constants required for the generation of the lowthrust phase, $R 2$ is the intermediate orbit (that is, at the end of the high-thrust phase) to initial orbit radius ratio and $e$ is the intermediate orbit eccentricity, the point at which the transfer switches from high to low-thrust. When the intermediate orbit is elliptical, the orbit ratio $R 2$ is the ratio of the intermediate orbit apogee to initial orbit radius. When the initial orbit is elliptical, the orbit ratio $R 2$ is the ratio of the intermediate orbit apogee to the initial orbit perigee.

The optimization parameters are subject to the following bounds

$$
\begin{aligned}
& W_{\sigma_{L}} \leq W_{\sigma} \leq W_{\sigma_{U}} \\
& R 2_{L} \leq R 2 \leq R 2_{U} \\
& e_{L} \leq e \leq e_{U}
\end{aligned}
$$

and the following active inequality constraints applied at each time-step,

$$
\begin{aligned}
& t-t_{M A X} \leq 0 \\
& \left|a_{t \text { arget }}-a\right| \leq 0.01 \\
& e-0.001 \leq 0 \\
& |i|-0.001^{\circ} \leq 0,
\end{aligned}
$$


where $t_{\text {MAX }}$ is the maximum allowable transfer time and is determined by the mission specification, $a_{\text {target }}$ is the semi-major axis for the final orbit, GEO in paper, which is used to scale the large semi-major axis value. It should be noted that no active equality constraints are applied.

The tolerances used in the optimization procedure apply to the objective function and inequality constraint parameters. The objective function tolerance is a bound on the change in the objective function, whilst the inequality constraint function is bounds and measures the magnitude of the constraint violation. A tolerance of $1 \times 10^{-9}$ was used for each. The optimization stops if the objective or function tolerances alone are satisfied, however it will not stop if the constraint function only is satisfied. The possibility then arises that the optimization will halt without the constraint function being satisfied.

\subsection{High-Thrust Phase}

It is assumed the high-thrust section is conducted through one or two impulsive burns; accounting for a circular or elliptical initial orbit respectively. This is based on the minimum energy Hohmann transfer. The first burn is used to enter the transfer orbit that (typically) takes the spacecraft beyond the target orbit. In the case where the lowthrust system is activated at the apogee of this orbit, i.e. the low-thrust phase begins with the eccentricity of the transfer orbit, this is the only high-thrust burn performed unless a plane change is required, and the transfer orbit becomes the intermediate orbit. In the case where the spacecraft enters an intermediate orbit at the transfer orbit apogee, a second high-thrust impulse, incorporating any plane change, is used to achieve this before the low-thrust system is activated. The high-thrust phase optimization involves two variables, the orbit ratio $R 2$ and the intermediate orbit eccentricity, $e$. The optimizer can vary $R 2$ to increases/decrease the intermediate orbit apogee in order to reduce the velocity requirement of the high-thrust phase. Additionally, the eccentricity, $e$, of this intermediate orbit can also be modified by the optimizer to lower the velocity requirement of the high-thrust phase.

When the high-thrust propulsion system performs the plane change, it is assumed that the orbit raise and plane change maneuvers are combined as this has been found to be more propellant effective. The plane change is distributed over two impulses; one at the initial/transfer orbit node and the second at the transfer/intermediate orbit node with the optimal split determined by numerically solving

$$
\sin s \Delta I=\frac{\Delta V_{i} v_{f} v_{\text {trans }} \sin ((1-s) \Delta I)}{\Delta V_{f} v_{i} v_{\text {trans }}},
$$

where $\Delta I$ is the total plane change, $s$ is the percentage plane change per maneuver, $\Delta V$ is velocity change, $v$ is the velocity change, and subscripts $i, f$ and trans, denote initial, final and transfer orbits. Note that this presumes the orbit major axis and line of nodes are equivalent. Finally note that the co-planar high-thrust phase still allows for a variable intermediate orbit.

\subsection{Low-Thrust Phase}

Locally optimal control laws can be used to generate complex trajectories with varying mission constraints. However, the effectiveness, and therefore optimality of these control laws, is often dependent on how they are combined or 'blended' to form a near-optimal thrust direction vector. In [45] a blending method known as ( $\left.\mathrm{A}^{\mathrm{n}} \mathrm{D}\right)$ was suggested for use in generating solar sail trajectories with a constrained thrust direction vector. It was proposed in that work that weighting constants be calculated as a function of the osculating elements, as opposed time; thus enabling the potential controller to account for unforeseen perturbations in the trajectory. This method calculated the weights for each control law based on the sail's time to target if it solely used that control law and its effective use of the sail. A user defined constant was also used to tailor the trajectories to suit certain mission specifications.

The method implemented herein is closely related to that of [45] but does incorporate some of the ideas discussed in $[42,43,58]$. It is adapted here to suit low-thrust technologies without the limitations of a sail i.e. the thrust can be directed in any direction as and when it is required. Firstly, the deficit (time to target) of each control 
law is calculated based on the maximized thrust vector if it were solely used and assuming a constant rate of change of the orbital elements. These rates of change are then normalized with respect to the largest; resultantly each control law receives a score between zero and one: zero meaning the control law has achieved its target and one meaning it is furthest, in terms of time, from its target value. The control laws, $\widehat{\lambda}$, are then multiplied by a constant, $W$, based on mission specification before finally being blended using the averaging technique

$$
\hat{\lambda}_{b}=\frac{\sum w_{\sigma} \tilde{\lambda}_{\sigma}}{\left\|\sum W_{\sigma} \hat{\lambda}_{\sigma}\right\|}
$$

where, $\sigma=a, e_{v} r_{p}, i$, subscript $b$ denotes blended, and $r_{p}$ is the radius of perigee. This forms the maximized thrust direction vector. Again, this method is dependent on a weighting constant that somehow needs to be determined. However, as this constant is independent of time and is specified at the beginning of the trajectory design, it can easily be determined in a non-intensive optimization procedure.

The low-thrust phase optimization involves a maximum of four variables, or weighting constants, however for the case where the high-thrust phase performs the plane change only three constants are required and are applied to the semi-major axis, eccentricity and radius of perigee control laws. The use of these constants reduces optimization complexity as each control law is prioritized before each trajectory calculation as opposed to each control law being prioritized at every time-step. It should be noted however that by implementing this method, a degree of accuracy is sacrificed as these constants are general for a whole trajectory as opposed to being variable to suit the spacecraft position at each individual time step.

\section{Geostationary Insertion}

The Geostationary Transfer Orbit, GTO, to GEO transfer parameters used are detailed in Table 1, and the spacecraft parameters in Table 2 for the use of QinetiQ T6 thruster(s), qualified up to a thrust of $145 \mathrm{mN}$ per thruster [59], and Table 3 for the use of SNECMA PPS ${ }^{\circledR} 5000$ thruster(s), where a thrust of $250 \mathrm{mN}$ is assumed per thruster [60]. Mission analysis considers the implication of operating the QinetiQ T6 thruster in a pair to aid orbit insertion, as well as the effect of switching this to a pair of SNECMA PPS ${ }^{\circledR} 5000$ thrusters. Note that a single SNECMA PPS ${ }^{\circledR} 5000$ thruster offers a similar thrust to the pair of QinetiQ T6 thrusters, but with a lower specific impulse, hence this case is not considered further.

\begin{tabular}{ll}
\hline Parameter (units) & Value \\
\hline Initial Orbit Perigee Altitude $(\mathrm{km})$ & 250 \\
Initial Orbit Perigee, $r_{i}(\mathrm{~m})$ & 6628100 \\
Initial Orbit Apogee Altitude (km) & 35945 \\
Initial Orbit Apogee, $r_{i}(\mathrm{~m})$ & 42359045 \\
Target Orbit Altitude (km) & 35945 \\
Target Orbit, $r_{t}(\mathrm{~m})$ & 42359045 \\
Initial orbit inclination (deg.) & 6.0 \\
Target orbit inclination (deg.) & 0.0 \\
Plane Change, $\Delta I$ (rad.) & 0.105 \\
\hline
\end{tabular}

Table 1 GTO - GEO orbit insertion specification for Ariane 5 from Kourou Europe Spaceport

\begin{tabular}{ll}
\hline Parameter (units) & Value \\
\hline High-Thrust System Specific Impulse (s) & 320 \\
Low-Thrust System Specific Impulse (s) & 4600
\end{tabular}


Critical Specific Impulse Ratio 14.375

Table 2 Spacecraft parameters with a QinetiQ T6 electric propulsion system

\begin{tabular}{ll}
\hline Parameter (units) & Value \\
\hline High-Thrust System Specific Impulse, (s) & 320 \\
Low-Thrust System Specific Impulse, (s) & 2180 \\
Critical Specific Impulse Ratio & 6.813 \\
\hline
\end{tabular}

Table 3 Spacecraft parameters with a SNECMA PPS ${ }^{2} 5000$ electric propulsion system

\subsection{0-day Transfer}

Analysis is presented in Figure 1 and Figure 2 for a GTO - GEO transfer with a 150-day duration and 290mN of electric propulsion thrust, assuming two QinetiQ T6 electric propulsion thrusters, with the mass inserted into GTO, the spacecraft wet mass, being varied from 2000 - $9100 \mathrm{~kg}$. The 150-day time limit was selected as a perceived upper-limit on the period an operator would be willing to tolerate between launch and commencing revenuegenerating operations due to the finance model employed by operators. It is seen that in comparison to a Hohmann transfer the combined high- and low-thrust transfer offers a reduction in total propellant mass in all the cases considered; with the high-thrust plane change offering the best propellant mass fraction in all cases. Additionally, for the low-thrust plane change the combined high- and low-thrust transfer offers the most benefit when the spacecraft wet mass is low; on a $2000 \mathrm{~kg}$ wet mass the reduction in total propellant mass is $521 \mathrm{~kg}$ (26\%), decreasing to a total propellant mass saving of $306 \mathrm{~kg}$ (3\%) on a $9100 \mathrm{~kg}$ wet mass. However, although the total propellant mass saving on the high-thrust plane change remains relatively constant, varying $\pm 10 \mathrm{~kg}$, the total propellant mass saving as a percentage of the wet mass also decreases as the wet mass is increased; from 27 - 6\% in the mass range considered.

Analysis is presented in Figure 3 and Figure 4 for a GTO - GEO transfer with a 150-day time limit and 145mN of electric propulsion thrust, assuming single QinetiQ T6 electric propulsion thrusters, with the mass inserted into GTO, the spacecraft wet mass, being varied over the same range as before. In general, the same trends are observed. However, it is noted that no meaningful result can be gained in the low-thrust plane change scenario beyond around $6000 \mathrm{~kg}$ due to the level of acceleration provided by the electric propulsion system. It was found that for a wet mass of $9100 \mathrm{~kg}$, a circular orbit at GEO radius requires 235 days to perform only the plane change maneuver.

The analysis presented is repeated for a 150-day transfer using a pair of SNECMA PPS $₫ 5000$ thrusters generating $500 \mathrm{mN}$ of thrust. The results are illustrated in Figure 5 and Figure 6. Broadly speaking the same general trends are observed. However, it is of note that despite the lower specific impulse the larger thrust provides an increased propellant mass saving in all cases when compared to the twin QinetiQ T6 thruster scenario.

\subsection{0-day Transfer}

The analysis is repeated herein for a 90-day transfer, and illustrated in Figure 7 and Figure 8 for the 290mN, and Figure 9 and Figure 10 for the $145 \mathrm{mN}$ case. The 90 -day transfer offers a potentially more tolerable period between launch and commencing revenue-generating operations as perceived from an operators finance model perspective. Broadly speaking the same general trends are observed. However, it is of note that for the $290 \mathrm{mN}$ case the maximum wet mass for the low-thrust plane change reduces to around $7500 \mathrm{~kg}$, and to around $3750 \mathrm{~kg}$ for the $145 \mathrm{mN}$ case.

The analysis is repeated for a 90-day transfer, using a pair of SNECMA PPS®5000 Thrusters generating 500mN of thrust, and illustrated in Figure 11 and Figure 12. Broadly speaking the same general trends are observed as before and once again, it is of note that despite the lower specific impulse the larger thrust provides an increased propellant mass saving in all cases when compared to the twin QinetiQ T6 thruster scenario. It is also noted that in comparison to the longer transfer time cases, a great propellant mass saving is achieved. 


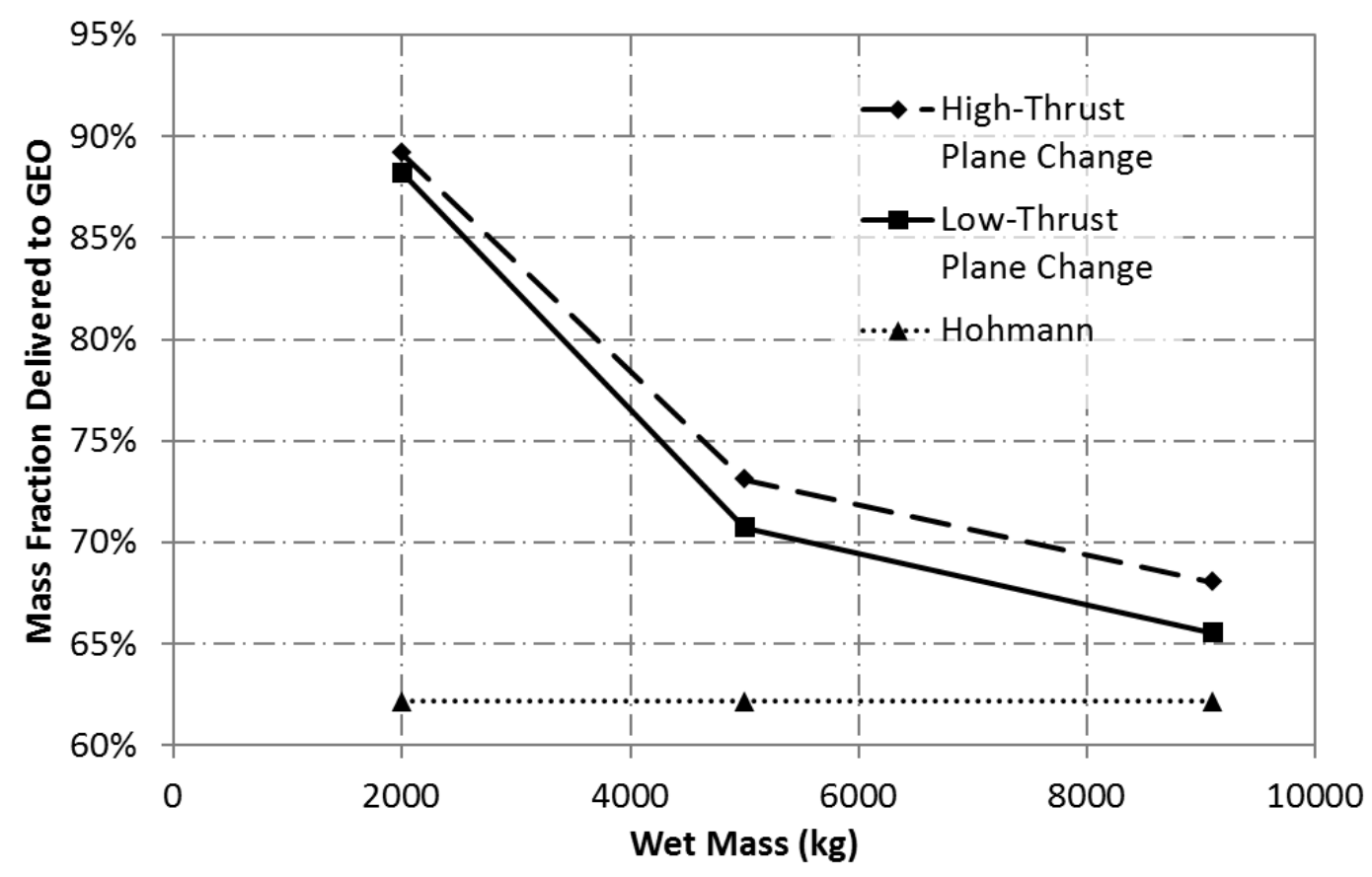

Figure 1 Mass fraction delivered to GEO from GTO with a 150-day time limit and $290 \mathrm{mN}$ of electric propulsion thrust

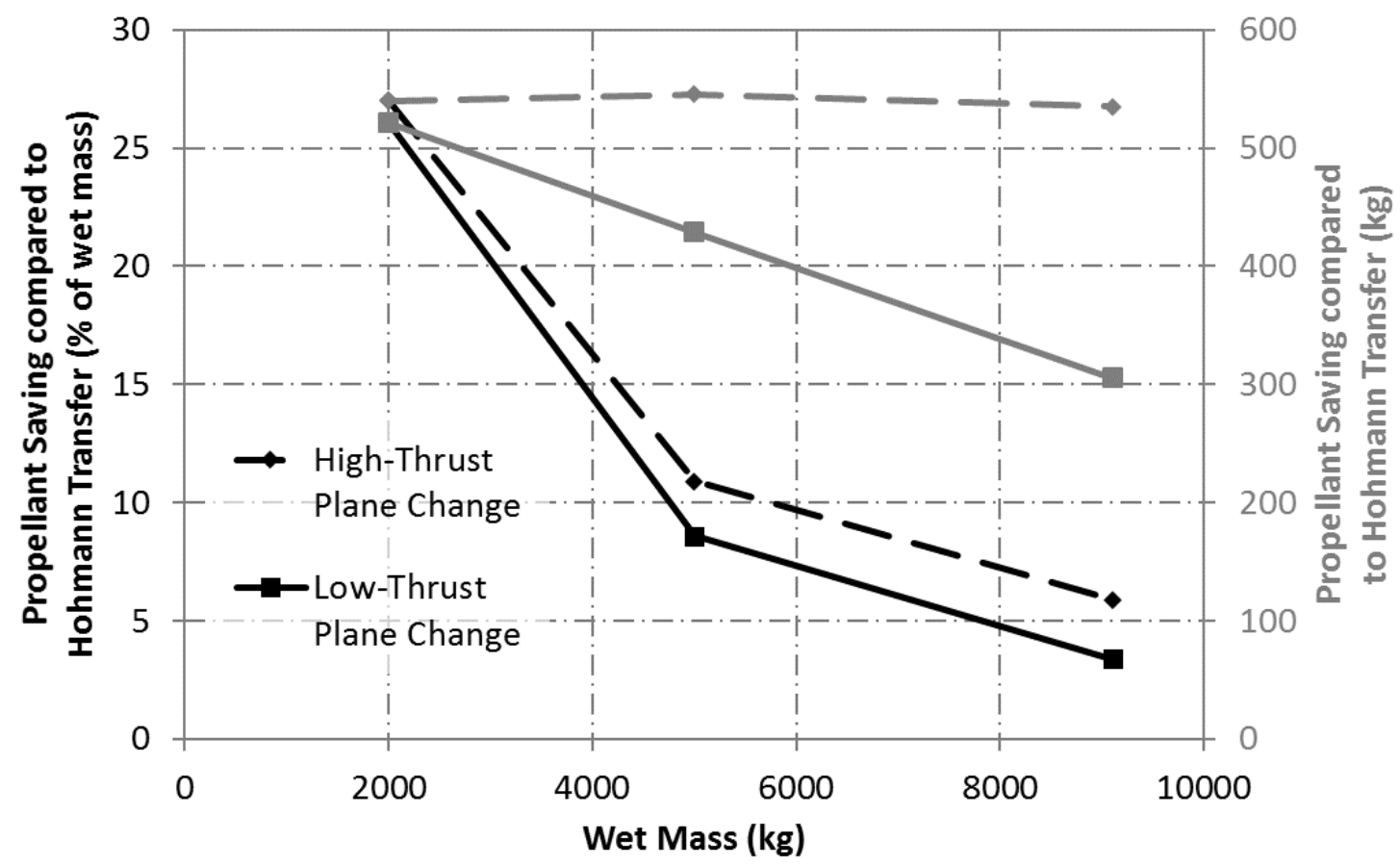

Figure 2 Propellant mass saving in comparison to a Hohmann transfer with a 150-day time limit and $290 \mathrm{mN}$ of electric propulsion thrust 


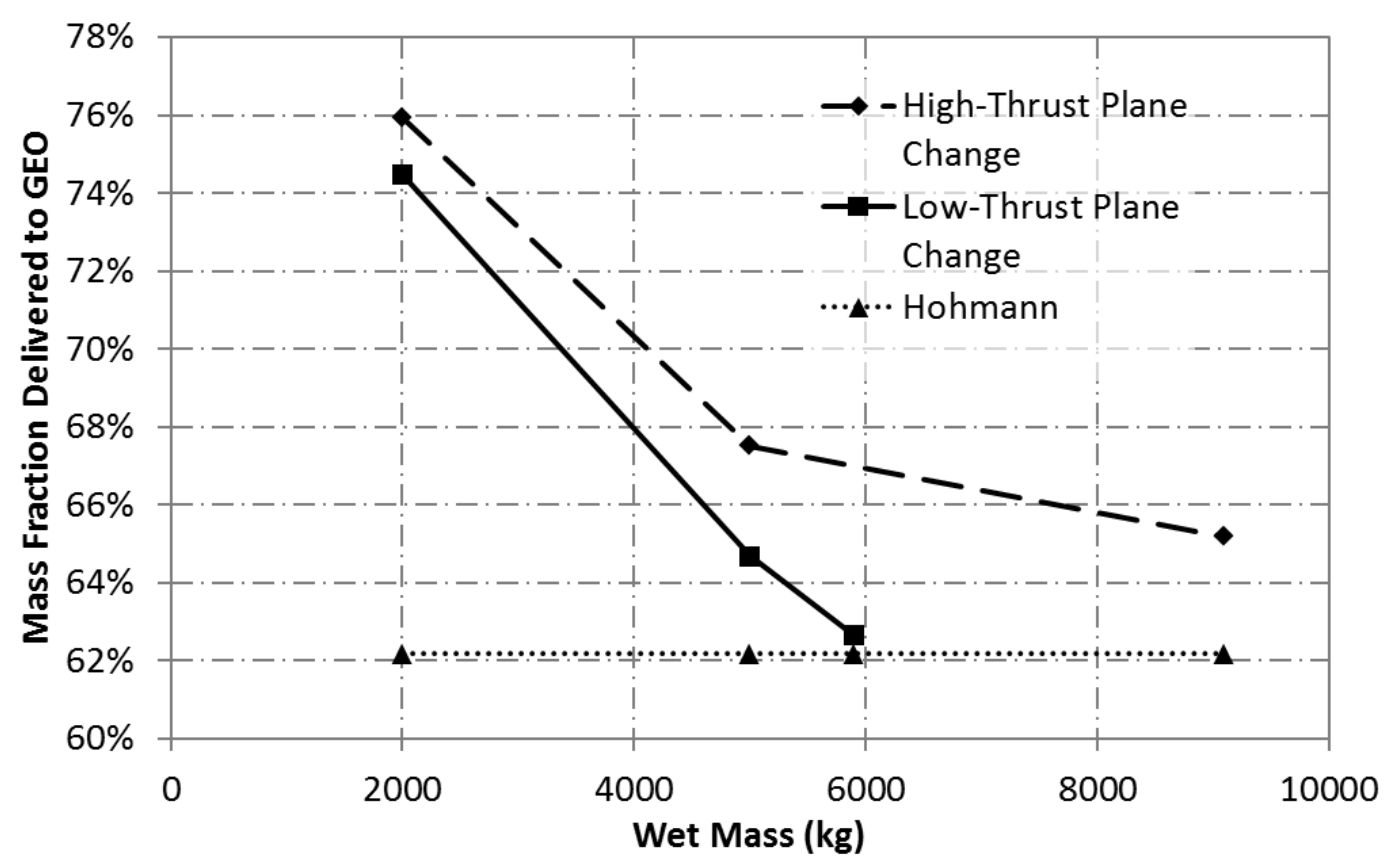

Figure 3 Mass fraction delivered to GEO from GTO with a 150-day time limit and $145 \mathrm{mN}$ of electric propulsion thrust

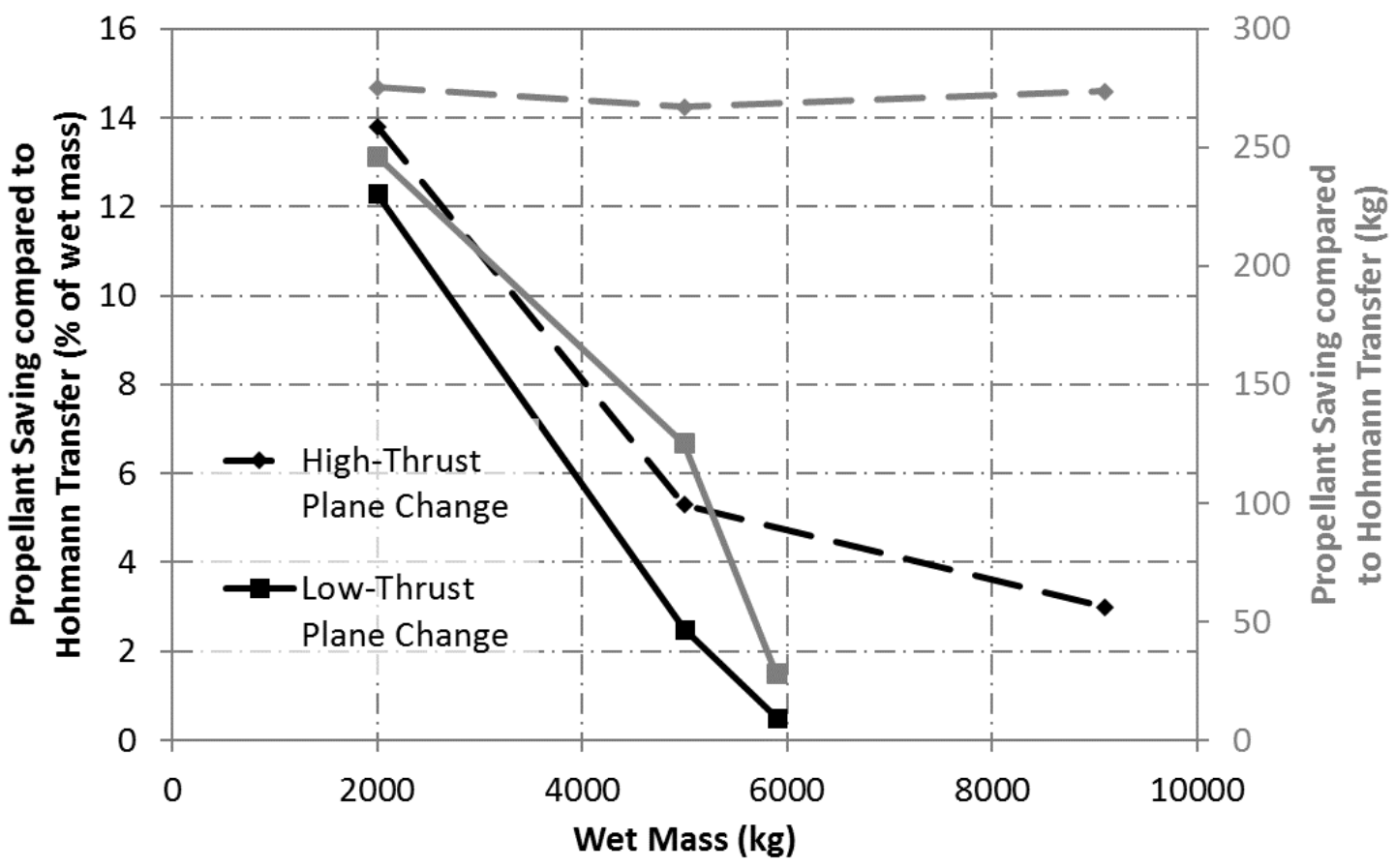

Figure 4 Propellant mass saving in comparison to a Hohmann transfer with a 150-day time limit and $145 \mathrm{mN}$ of electric propulsion thrust 


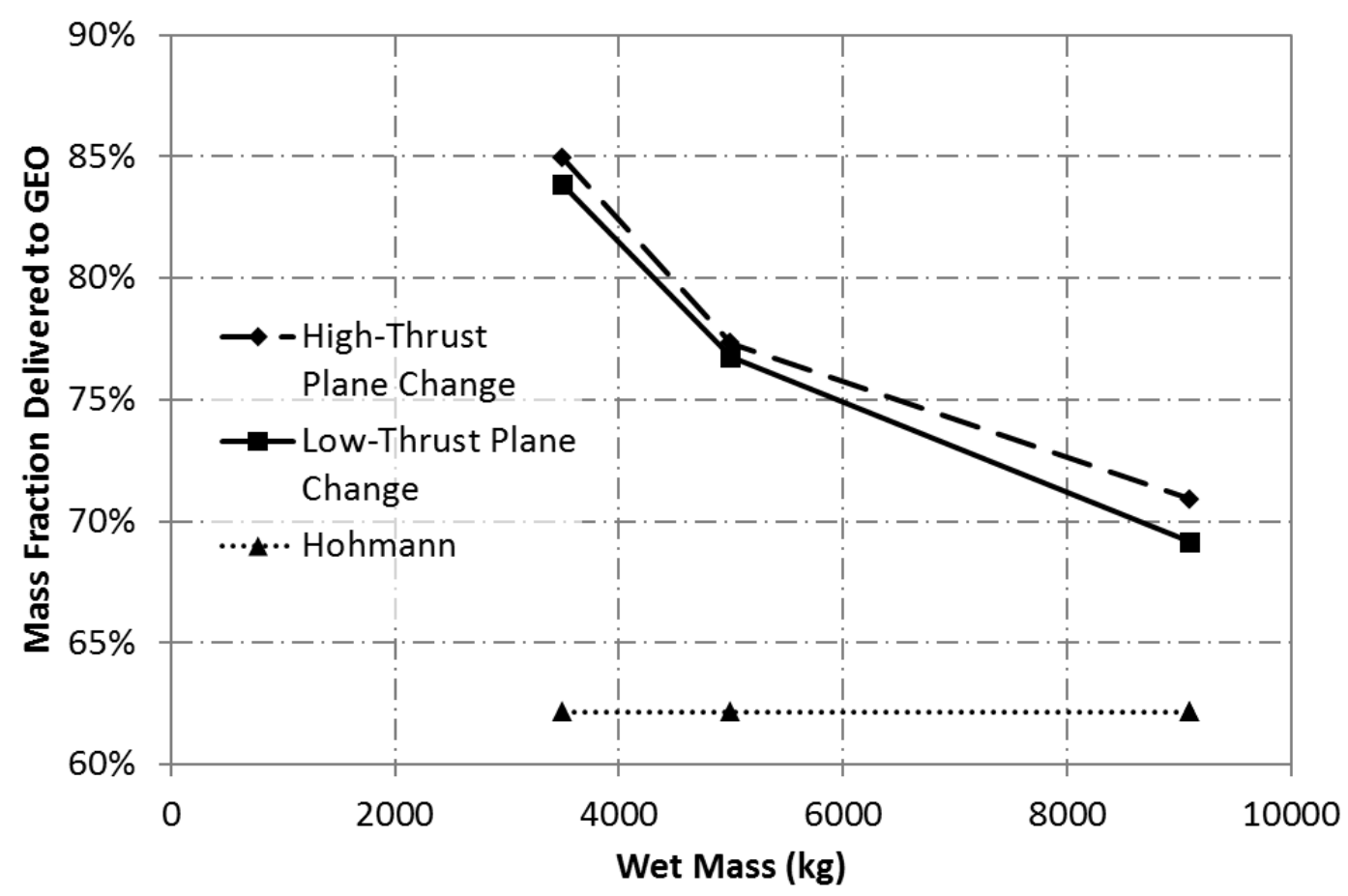

Figure 5 Mass fraction delivered to GEO from GTO with a 150-day time limit and $500 \mathrm{mN}$ of electric propulsion thrust

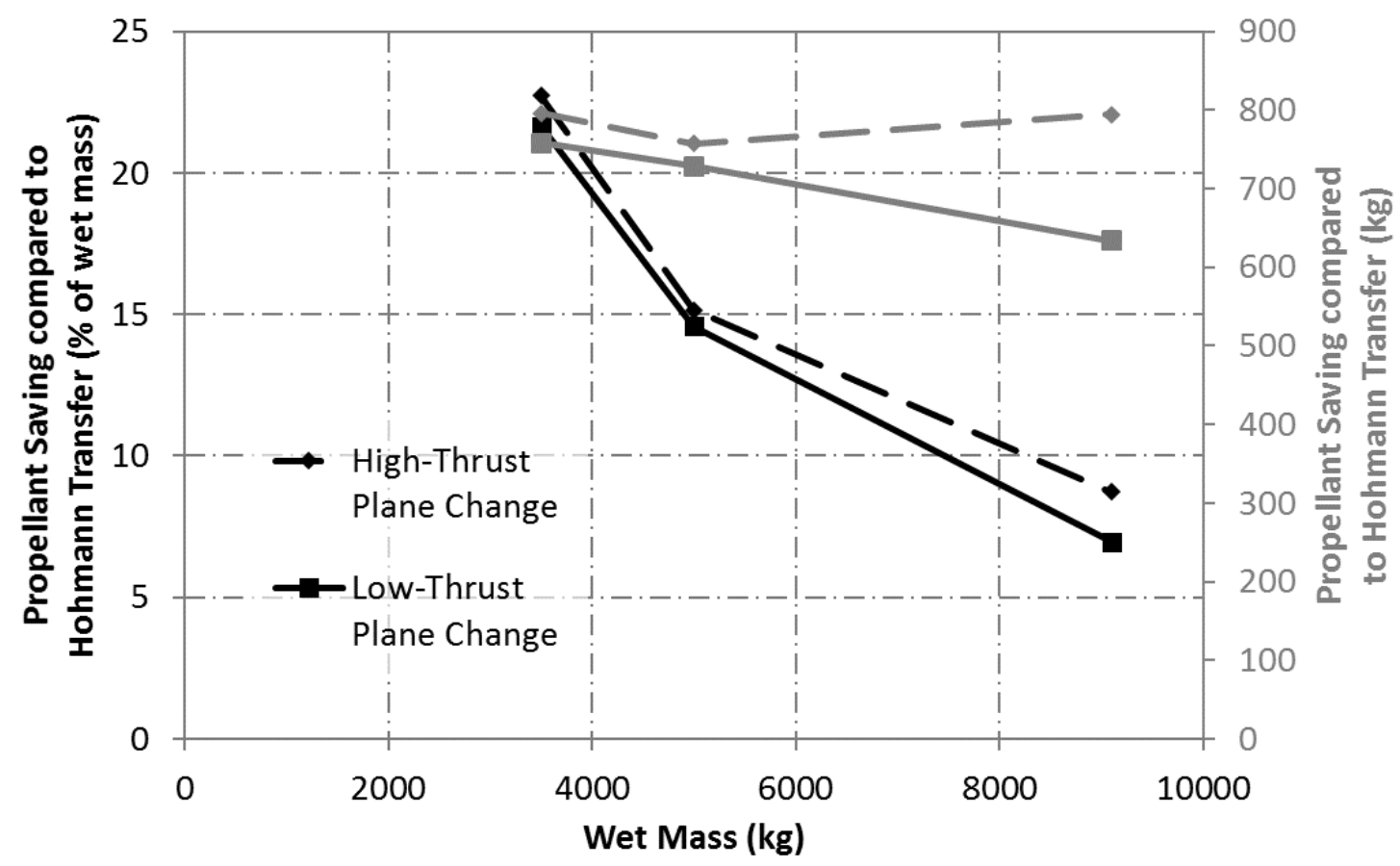

Figure 6 Propellant mass saving in comparison to a Hohmann transfer with a 150-day time limit and $500 \mathrm{mN}$ of electric propulsion thrust 


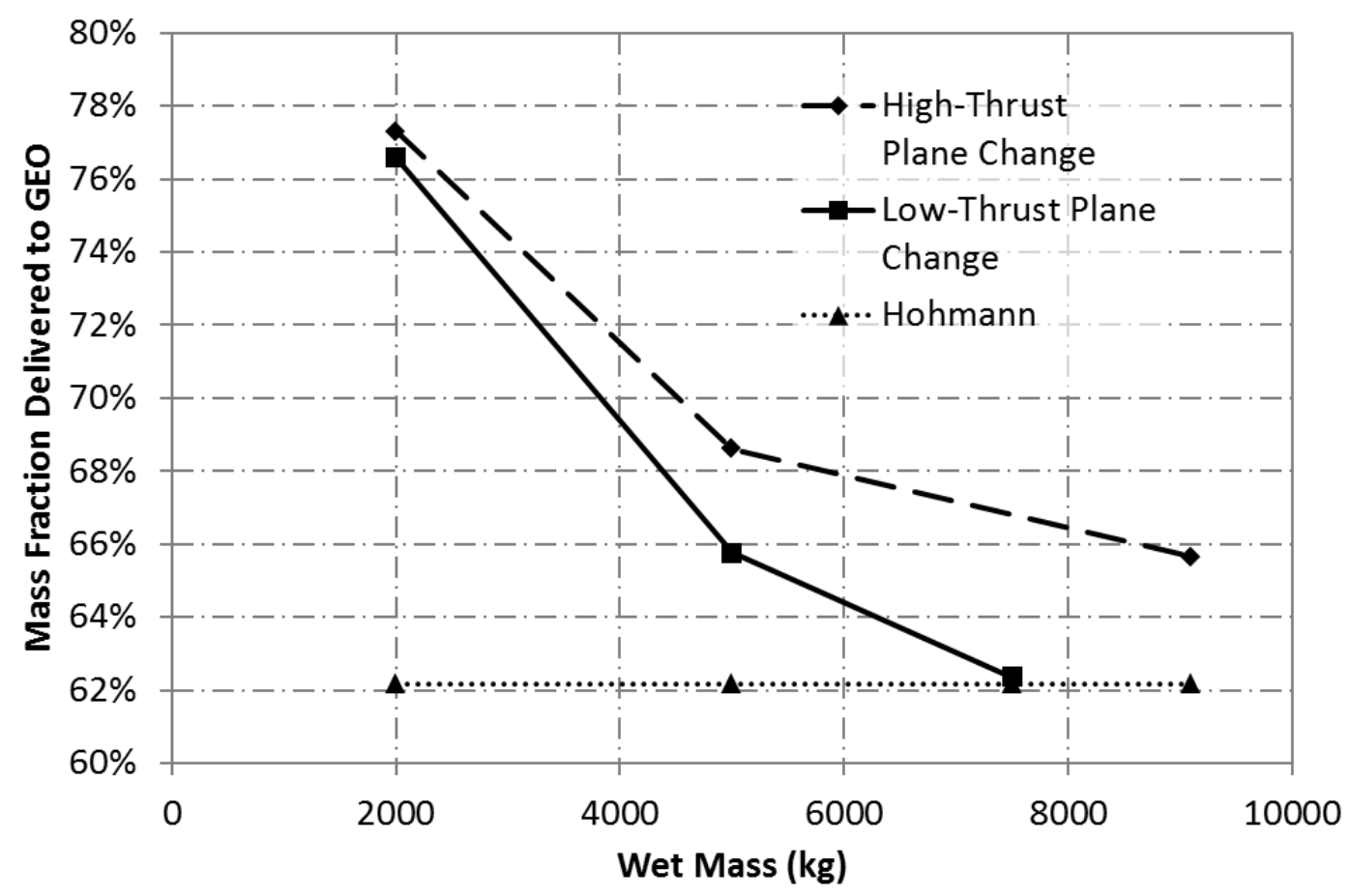

Figure 7 Mass fraction delivered to GEO from GTO with a 90-day time limit and $290 \mathrm{mN}$ of electric propulsion thrust

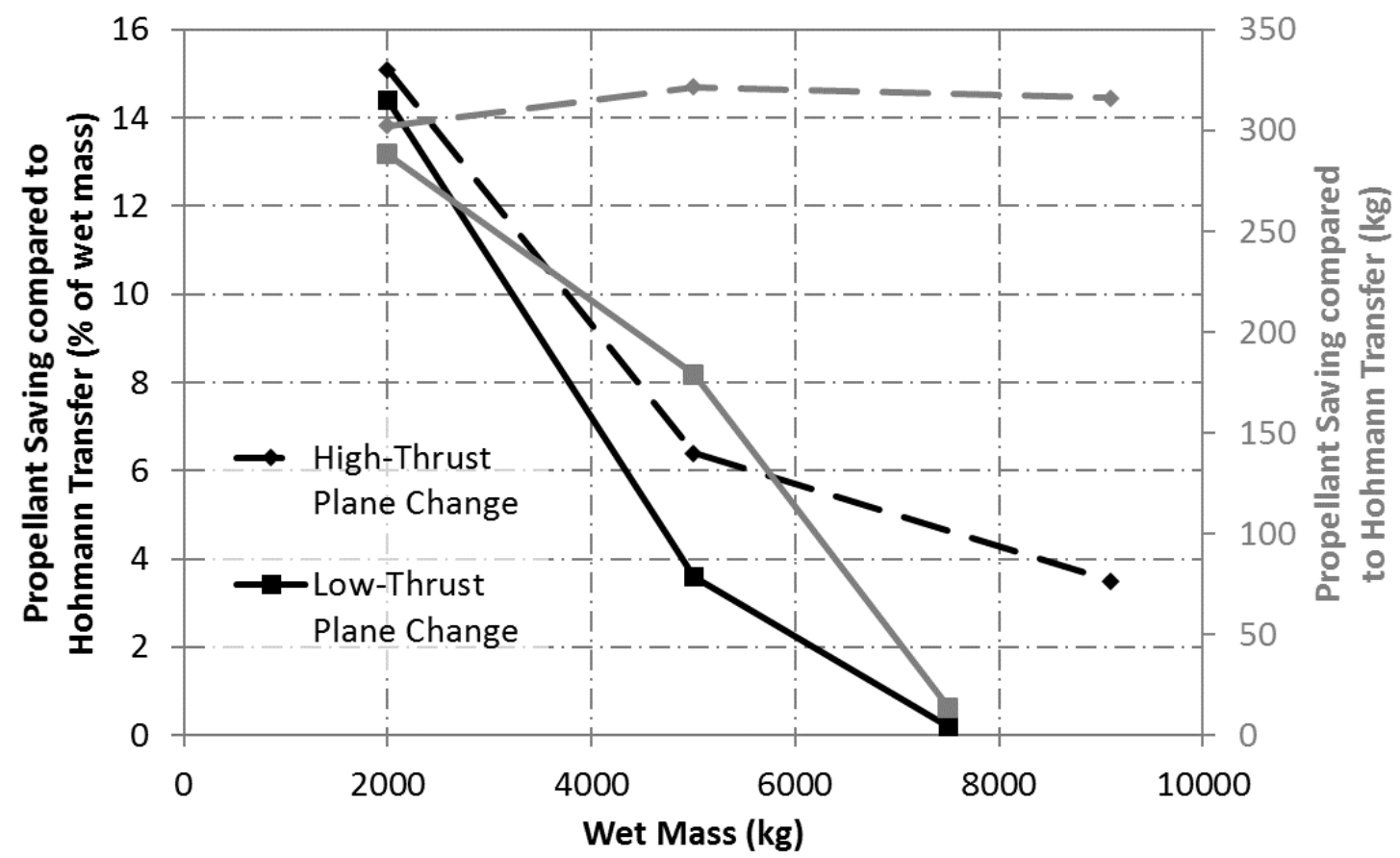

Figure 8 Propellant mass saving in comparison to a Hohmann transfer with a 90-day time limit and $290 \mathrm{mN}$ of electric propulsion thrust 


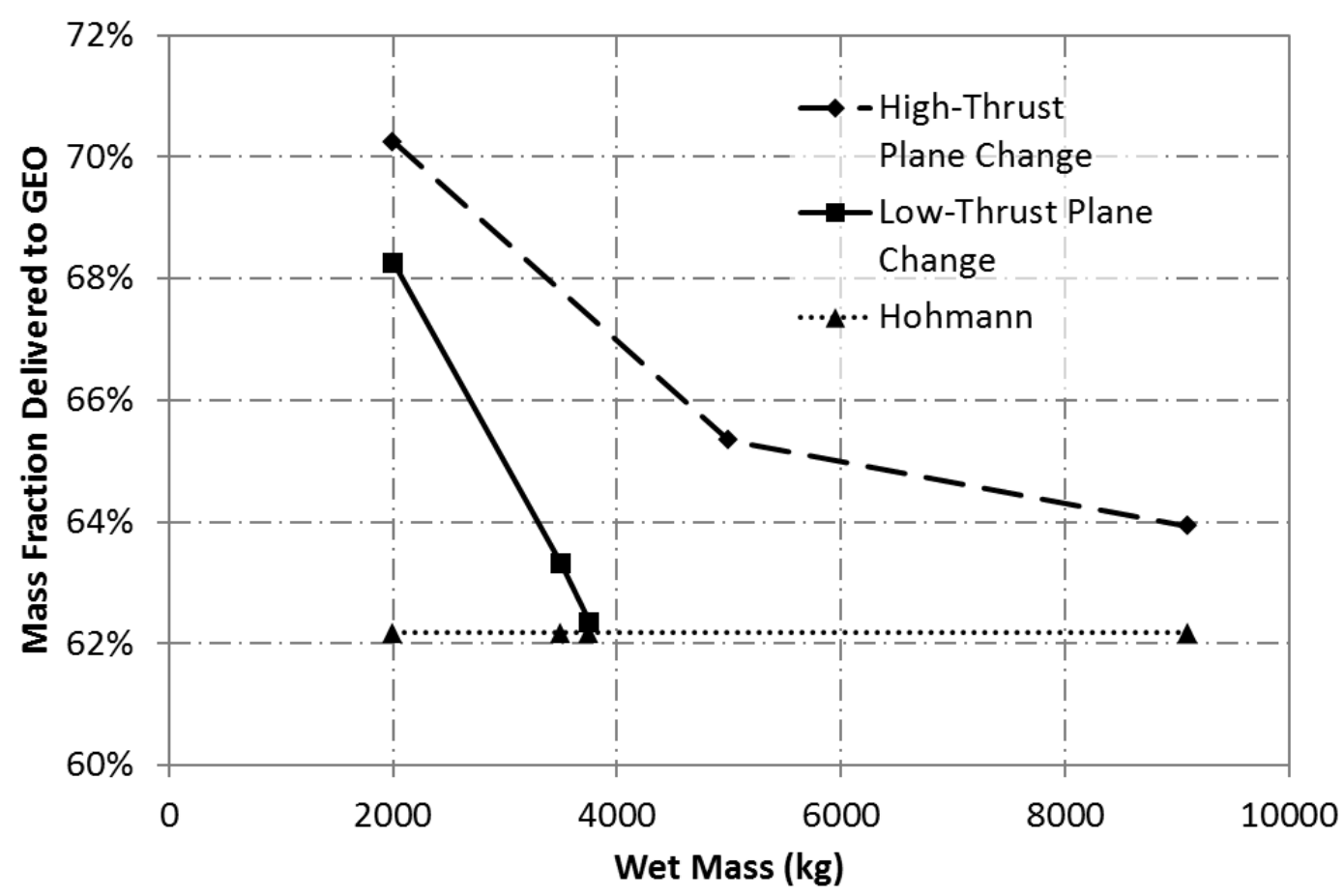

Figure 9 Mass fraction delivered to GEO from GTO with a 90-day time limit and $145 \mathrm{mN}$ of electric propulsion thrust

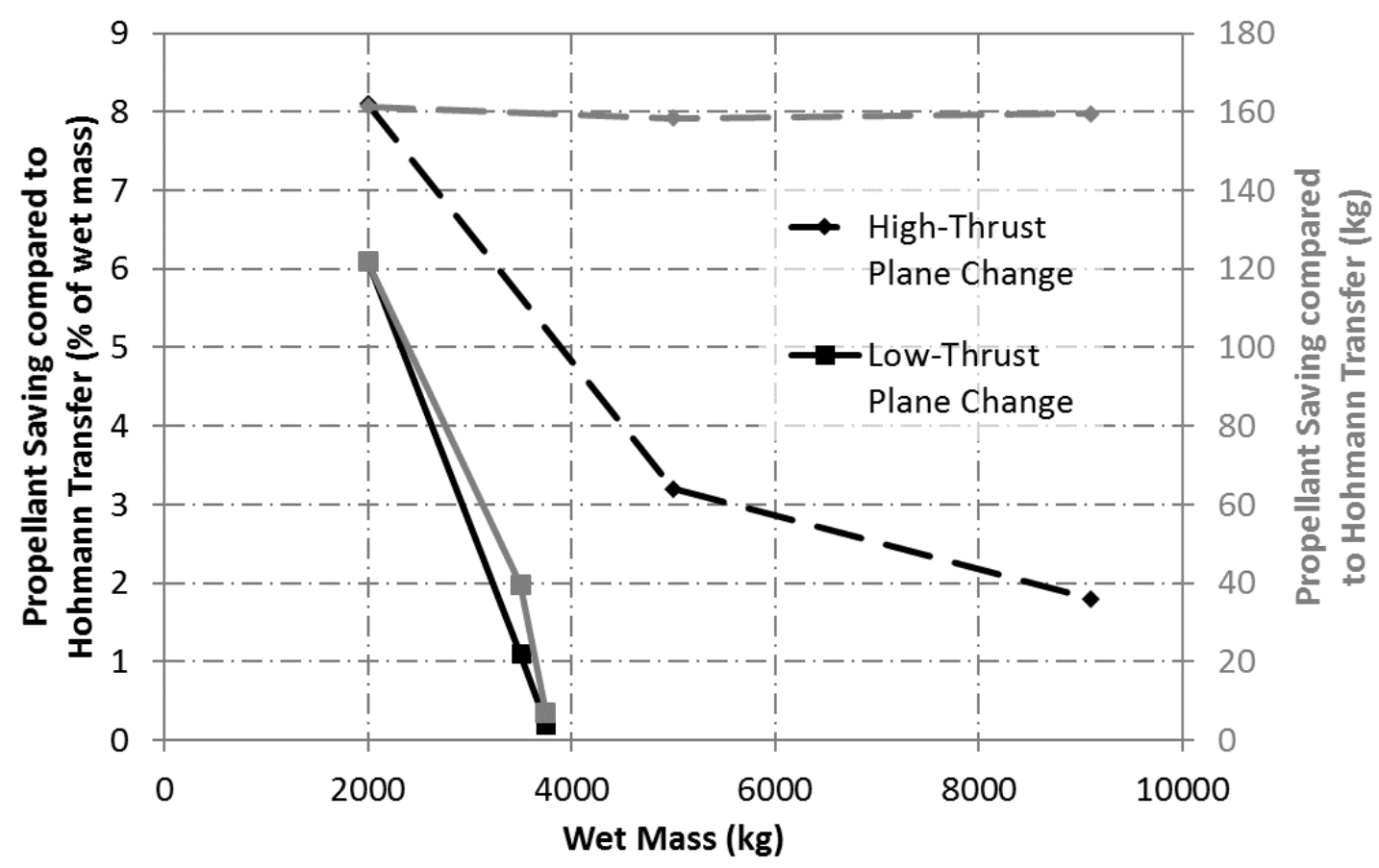

Figure 10 Propellant mass saving in comparison to a Hohmann transfer with a 90-day time limit and $145 \mathrm{mN}$ of electric propulsion thrust 


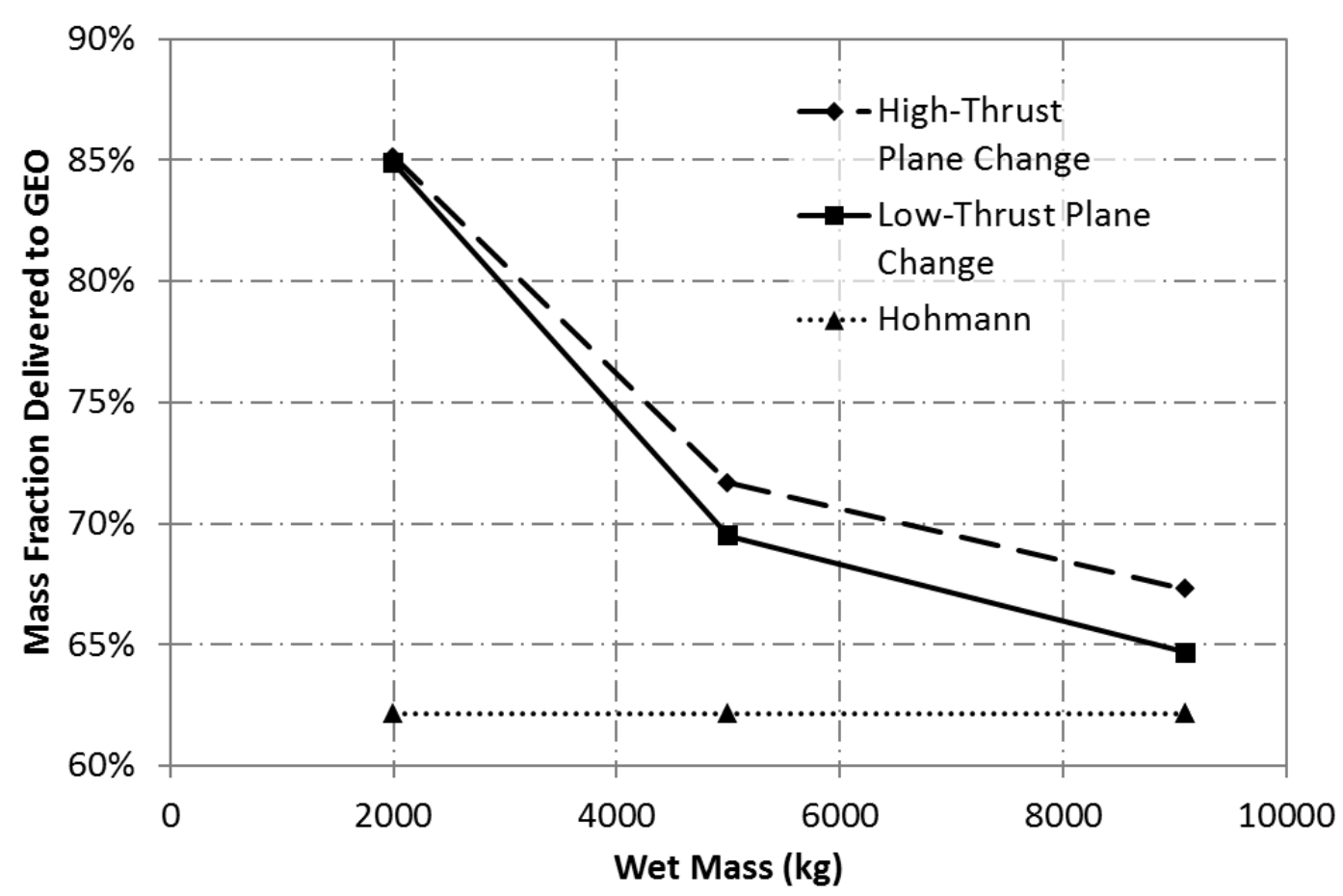

Figure 11 Mass fraction delivered to GEO from GTO with a 90-day time limit and $500 \mathrm{mN}$ of electric propulsion thrust

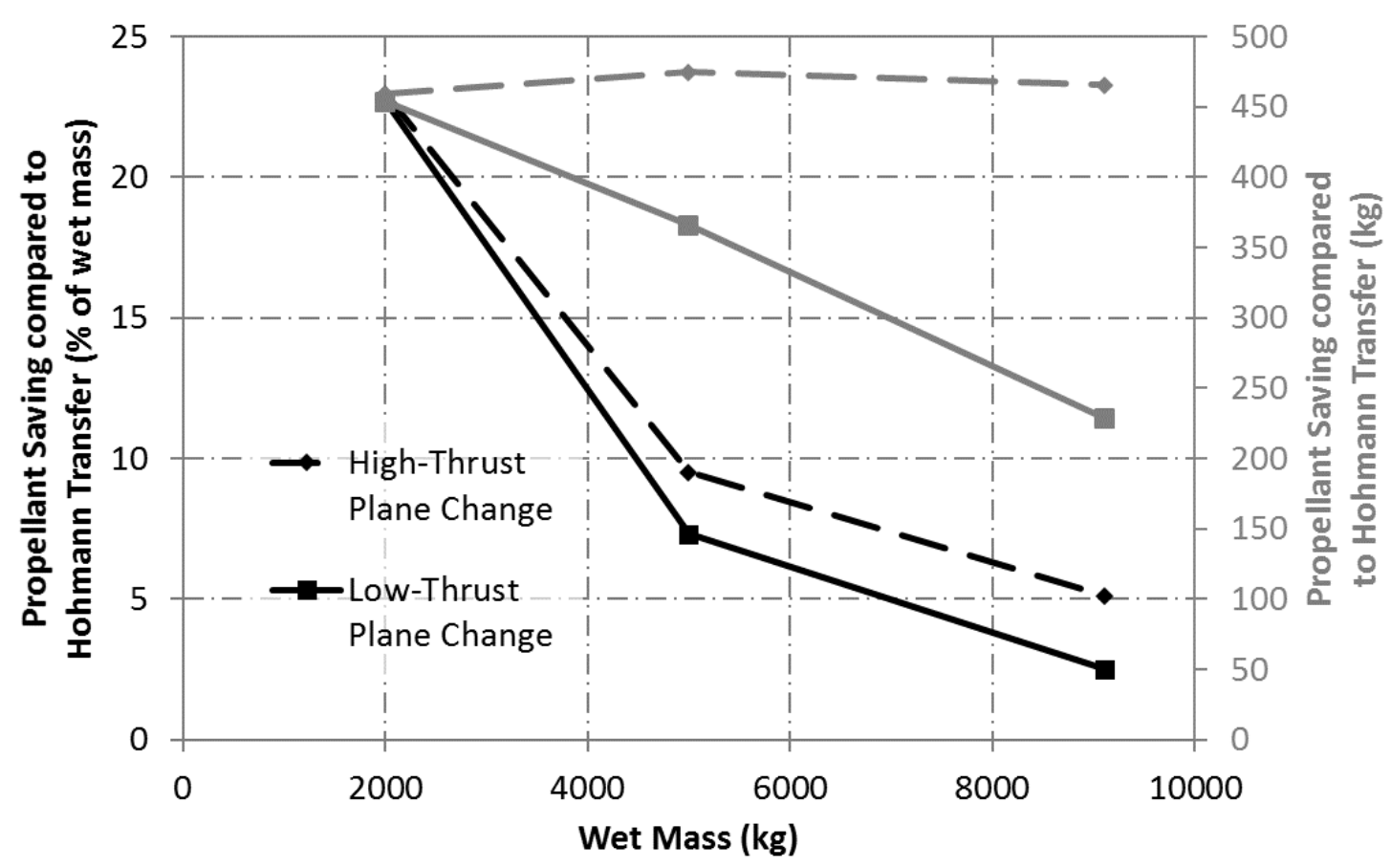

Figure 12 Propellant mass saving in comparison to a Hohmann transfer with a 90-day time limit and $500 \mathrm{mN}$ of electric propulsion thrust 


\subsection{Radiation Constraint}

The trapped radiation belts surrounding the Earth, often termed the Van Allen belts, consist of electrons and ions (predominantly protons) with energies ranging from the thermal $(<1 \mathrm{eV})$ to highly relativistic (tens of $\mathrm{MeV}$ for electrons and $\mathrm{GeV}$ for protons [61]. The important components from a spacecraft design perspective are the electrons with energies between $500 \mathrm{keV}$ to a few $\mathrm{MeV}$, and protons with energies $>100 \mathrm{MeV}$ due to their effect on spacecraft electronics [62,63]. The electrons are found in two toroidal regions, with latitudes up to around 60 degrees from the geomagnetic equator, centered on radii of approximately 1.3 Earth radii and 5 Earth radii, whilst the protons are focused on the inner of these two regions [62]. Note that the geomagnetic equator is displaced from the equator by approximately 10 degrees depending on the reference epoch.

Using the NASA AE-8/AP-8 radiation models and neglecting the effects of orbit inclination or geomagnetic latitude the simplified particle flux model shown in Figure 13 was derived and verified against the SPENVIS tool [64]. Using this model, a further active inequality constraint was added to the optimization process to maintain the radiation dose experienced throughout the transfer trajectory below a user determined value; radiation dose is defined as the cumulative or time-integral particle fluxes shown in Figure 13. Figure 14 shows the effect of doing so on the required propellant mass. In the unconstrained case, delivering the largest propellant reduction within the 90day transfer limit, the electron flux is almost 280 times that of a Hohmann transfer from GTO - GEO. However, the proton flux is almost identical. Note that the spacecraft is assumed to remain in GTO for three days for commissioning prior to both transfers being initiated. By reducing the additional electron flux incurred due to the use of low-thrust propulsion to seven times greater than that of the original Hohmann transfer from GTO - GEO an extra $231 \mathrm{~kg}$ of propellant is required, reducing the propellant mass saving over a Hohmann transfer from GTO GEO from $6.4 \%$ to $1.8 \%$; that is $321 \mathrm{~kg}$ down to $90 \mathrm{~kg}$. Notably, the proton flux in this scenario remains largely unchanged, with a reduction of just over $1 \%$.

As anticipated, it is seen in Figure 15 that as the electron flux through the low-thrust propelled trajectory is decreased the high-thrust propulsion system is used to increasingly maneuver beyond the radiation belts, whilst the low-thrust propulsion system is used to return to GEO. Note that for the unconstrained case the semi-major axis is 0.82 GEO radii at the end of the high-thrust phase, whilst it is 1.26 GEO radii for the lowest electron flux case. Meanwhile, no similar trend is observed in the variation of the eccentricity as the electron flux is decreased, although for the lowest electron flux case the eccentricity at the end of the high-thrust phase is the lowest. Figure 16 further illustrates the effect of limiting the electron flux, showing that both the radius of perigee and apogee at the end of the high-thrust phase increase as the electron flux is reduced. These results suggest that had a more complete radiation model been applied than that shown in Figure 13, the transfer trajectory is likely to exhibit a highly similar structure. Of note, as the additional electron flux incurred due to the use of low-thrust propulsion is reduced to a level around 90 times that of a Hohmann transfer from GTO - GEO, the radius of perigee begins to peak above the GEO radius, before reducing to the target. As such it would be viable to consider jettisoning the high-thrust propulsion system, including the tanks, into the graveyard region above GEO to reduce the spacecraft mass, and hence further increase the efficiency of the remaining low-thrust system, without contravening space debris guidelines [39,40]. 
Author Proof

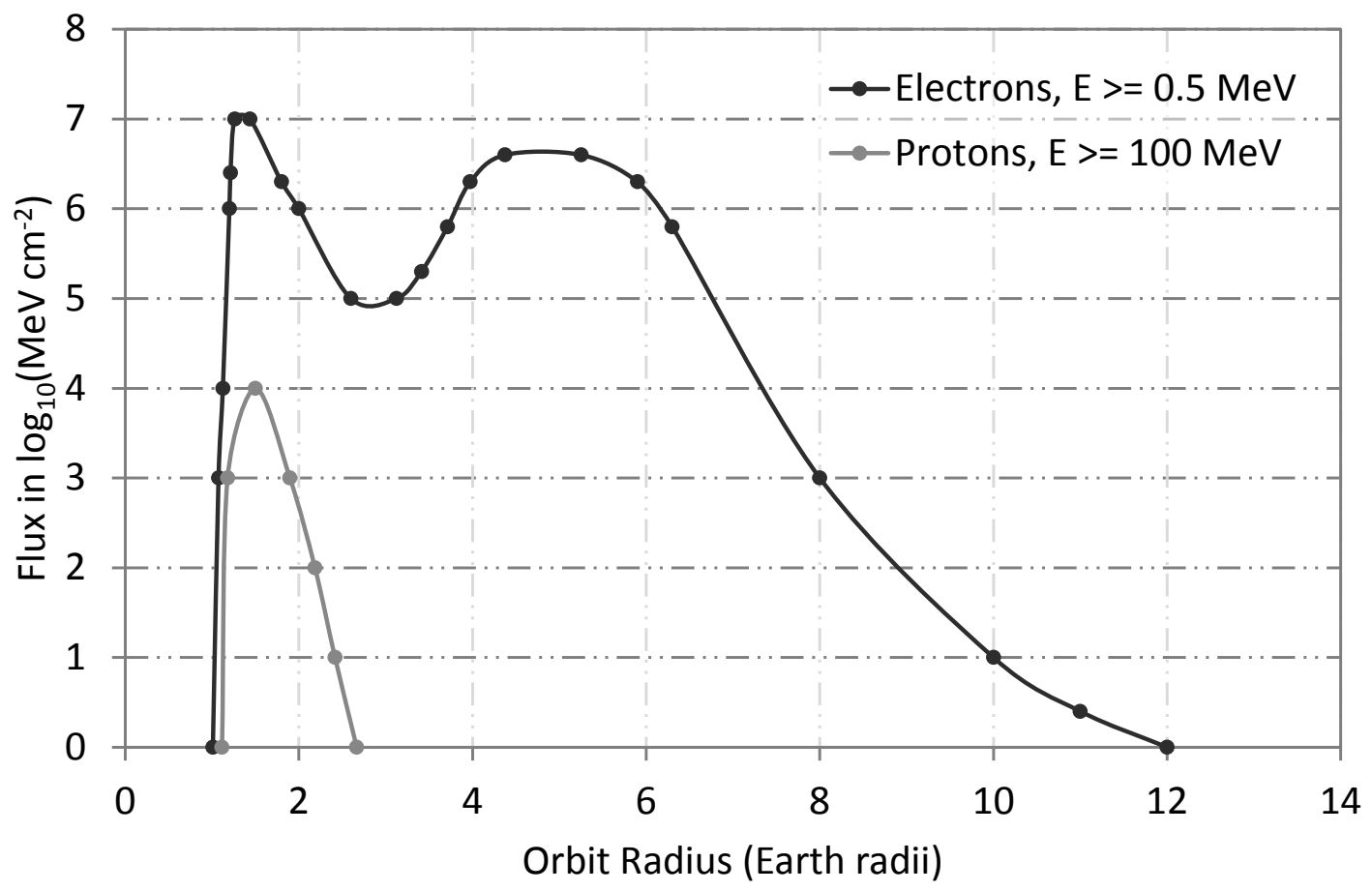

Figure 13 Integral omnidirectional electron and proton fluxes 


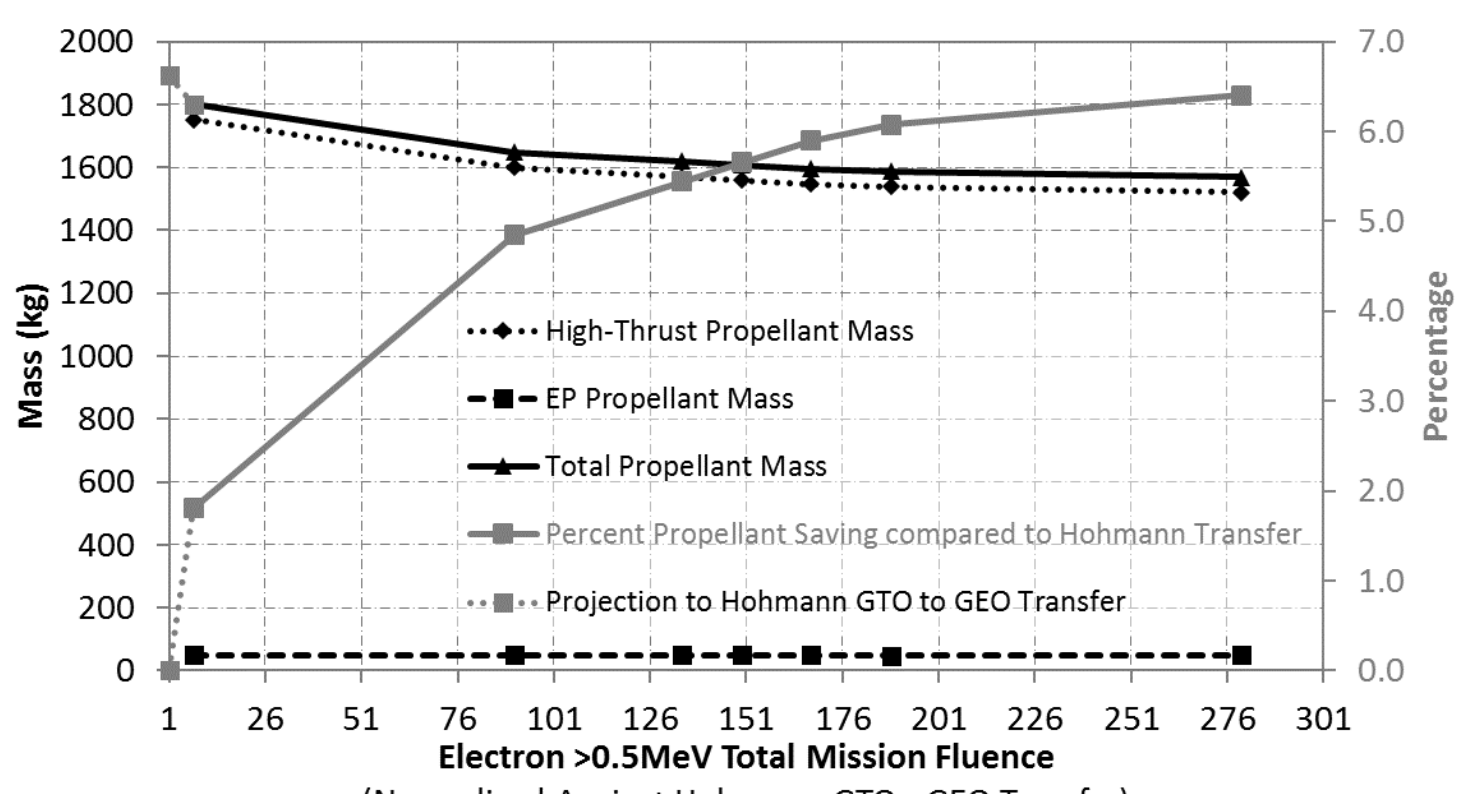

(Normalised Against Hohmann GTO - GEO Transfer)

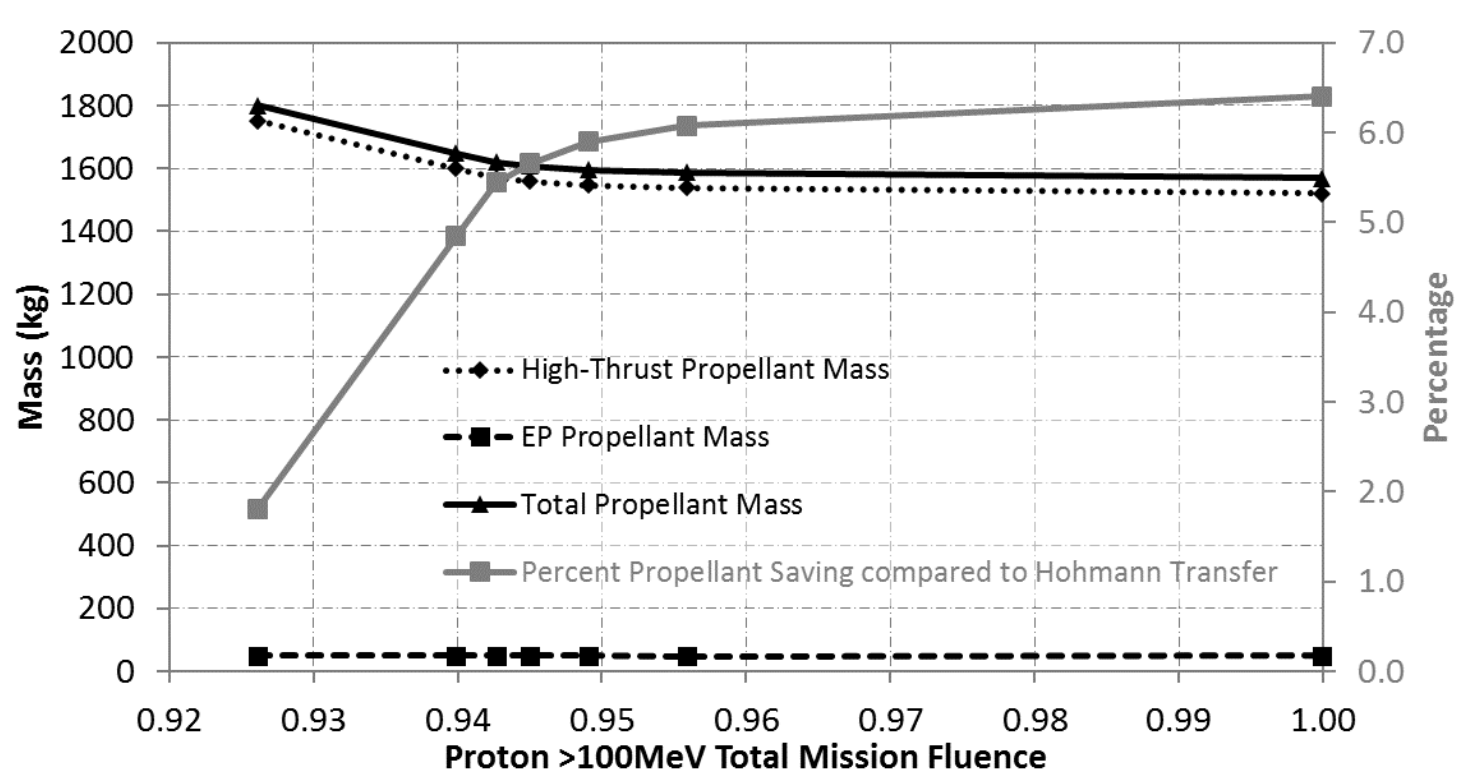

(Normalised Against Hohmann GTO - GEO Transfer)

Figure 14 Variation in propellant mass for a $5000 \mathrm{~kg}$ wet mass, 90 day, $290 \mathrm{mN}$ low-thrust transfer against the total electron (top) and proton (bottom) flux normalised against Hohmann GTO - GEO transfer 

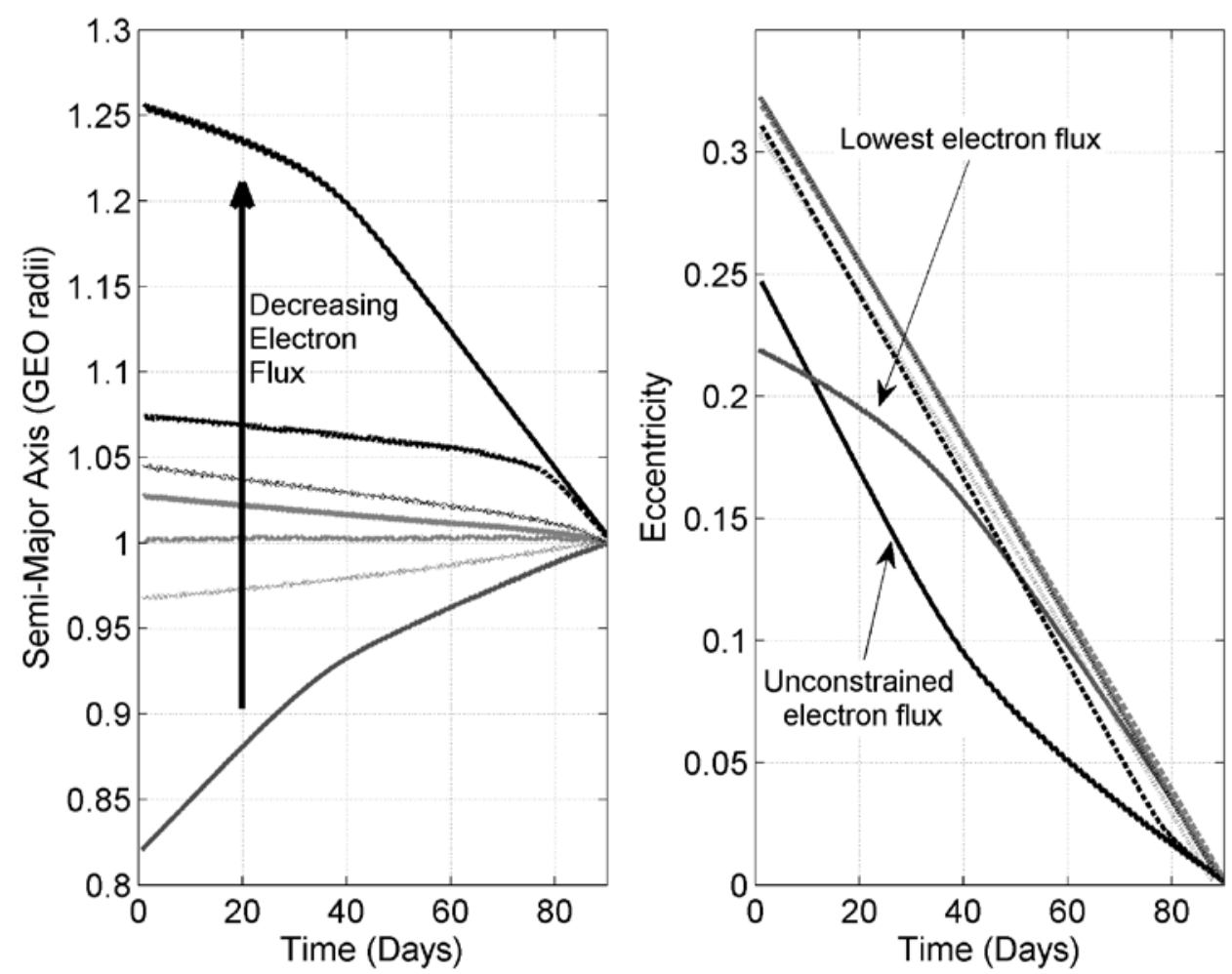

Figure 15 Semi-major axis and eccentricity variation with varying electron flux, each contour corresponds to a data point in Figure 14
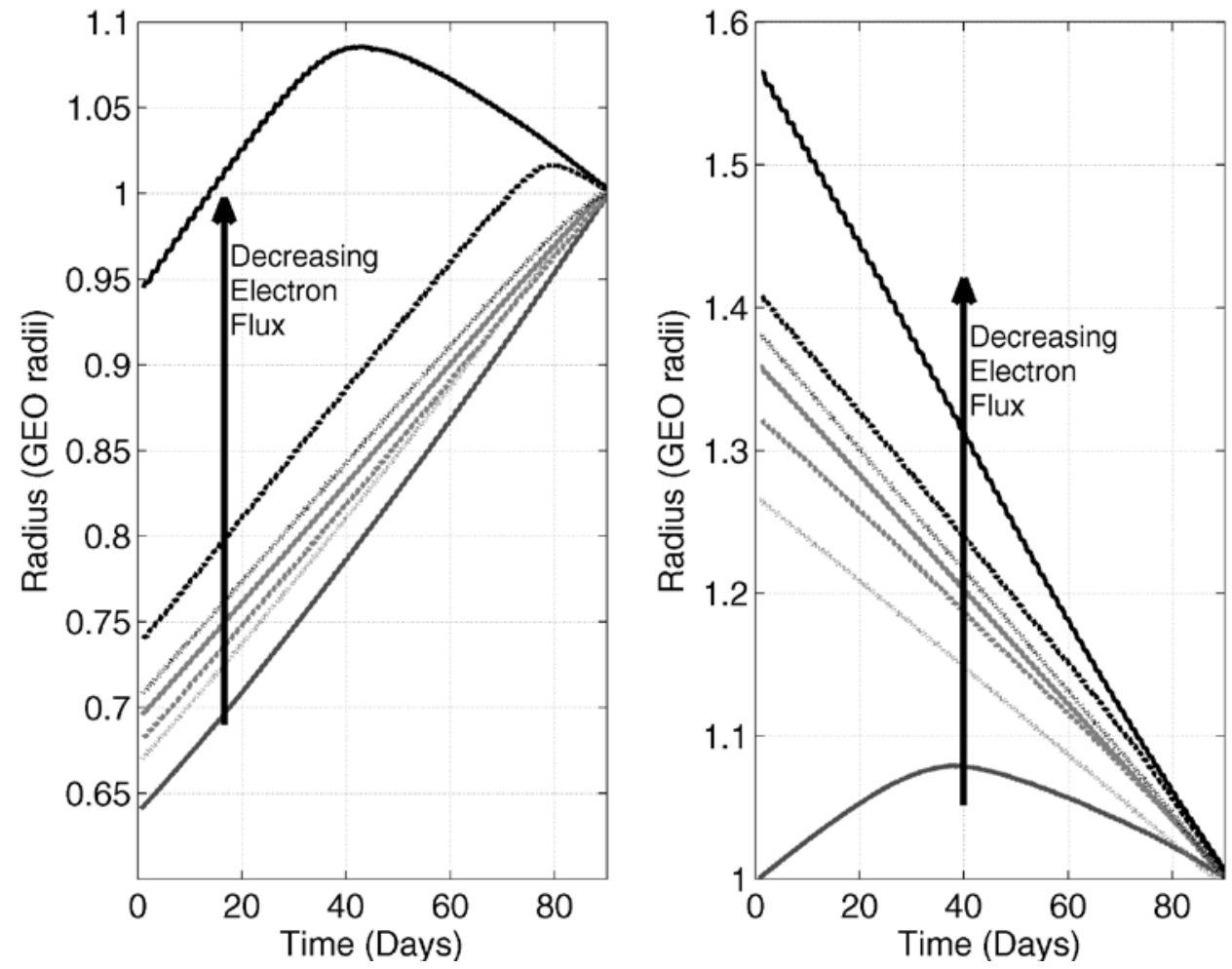

Figure 16 Radius of perigee (left) and apogee (right) variation with varying electron flux, each contour corresponds to a data point in Figure 14 


\section{Conclusion}

The sequential use of high and low-thrust propulsion for transfer from geostationary transfer orbit, GTO, to a geostationary orbit, GEO, was found to offer a combined propellant mass saving when compared with an equivalent high-thrust only transfer. This propellant mass saving was seen to increase as the allowable transfer duration is increased, and as the thrust from the low-thrust system is increased, assuming constant specific impulse. It was found that the required plane change maneuver is most propellant-efficiently performed by the high-thrust system. The propellant mass saving in such high-thrust plane change transfers varies little as the wet mass, i.e. the mass inserted into GTO, is increased. Consequently, the percentage benefit over the equivalent high-thrust only transfer decreases as wet mass is increased. Finally, it was shown that the propellant optimal trajectory incurs a non-minor increase in electron flux (for energies $>0.5 \mathrm{MeV}$ ) when compared to a Hohmann transfer from GTO to GEO, whilst the proton flux (for energies $>100 \mathrm{MeV}$ ) is almost identical. However, the electron flux can be reduced to a similar order of magnitude to that of the Hohmann transfer from GTO to GEO by increasing the high-thrust propellant consumption, whilst still delivering an improved mass fraction to GEO. Of note, the perigee in such scenarios increases beyond GEO before returning to the target value. Therefore, it would be viable to consider jettisoning the high-thrust propulsion system, including the tanks, into the graveyard region above GEO to reduce the spacecraft mass, and hence increase the efficiency of the remaining low-thrust system, without contravening space debris guidelines.

\section{Acknowledgments}

This project has received funding from the European Union's Horizon 2020 research and innovation programme under grant agreement No 634534. The authors thank Christoper Lowe, of the Strathclyde Space Institute at the University of Strathclyde, for his assistance in running some of the simulation cases.

\section{References}

[1] L.L. Sackett, H.L. Malchow, T.N. Edelbaum, Solar Electric Geocentric Transfer With Attitude Constraints: Analysis, NASA, 1975. http://trajectory.grc.nasa.gov/tools/sepspot/CR-134927V1.pdf.

[2] J. V. BreakwellL, D.C. Redding, Optimal low-thrust transfers to synchronous orbit, J. Guid. Control. Dyn. 7 (1984) 148-155. doi:10.2514/3.8560.

[3] W.A. Scheel, B.A. Conway, Optimization of very-low-thrust, many-revolution spacecraft trajectories, J. Guid. Control. Dyn. 17 (1994) 1185-1192. doi:10.2514/3.21331.

[4] Y. Matogawa, Optimum low thrust transfer to geosynchronous orbit, Acta Astronaut. 10 (1983) 467-478. doi:10.1016/0094-5765(83)90019-X.

[5] W.A. Scheel, B.A. Conway, Optimization of very-low-thrust, many-revolution spacecraft trajectories, J. Guid. Control Dyn. 17 (1994) 1185-1192. doi:10.2514/3.21331.

[6] D.B. Spencer, R.D. Culp, Designing continuous-thrust low-Earth-orbit to geosynchronous-Earth-orbit transfers, J. Spacecr. Rockets. 32 (1995) 1033-1038. doi:10.2514/3.26726.

[7] J. Kechichian, Optimal low-earth-orbit-geostationary-earth-orbit intermediate acceleration orbit transfer, J. Guid. Control Dyn. 20 (1997) 803-811. doi:10.2514/2.4116.

[8] J. Kechichian, Reformulation of Edelbaum's low-thrust transfer problem using optimal control theory, J. Guid. Control. Dyn. 20 (1997) 988-994. doi:10.2514/2.4145.

[9] C. Kluever, S. Oleson, Direct approach for computing near-optimal low-thrust earth-orbit transfers, J. Spacecr. Rockets. 35 (1998) 509-515. doi:10.2514/2.3360.

[10] C. Kluever, Geostationary orbit transfers using solar electric propulsion with specific impulse modulation, J. Spacecr. Rockets. 41 (2004) 461-466. doi:10.2514/1.10939.

[11] T. Haberkorn, P. Martinon, J. Gergaud, Low thrust minimum-fuel orbital transfer: a homotopic approach, J. Guid. Control. .... 27 (2004) 1046-1060. doi:10.2514/1.4022.

[12] Y. Gao, Near-optimal very low-thrust earth-orbit transfers and guidance schemes, J. Guid. Control. Dyn. 30 
(2007) 529-539. doi:10.2514/1.24836.

[13] L. Casalino, G. Colasurdo, Improved Edelbaum's approach to optimize low earth/geostationary orbits lowthrust transfers, J. Guid. Control. Dyn. 30 (2007) 1504-1510. doi:10.2514/1.28694.

[14] T. Edelbaum, The use of high-and low-thrust propulsion in combination for space missions, J. Astronaut. Sci. 9 (1962) 49-60.

[15] E. Choueiri, A critical history of electric propulsion: the first 50 years (1906-1956), J. Propuls. Power. (2004).

[16] W. Fimple, An Improved Theory of the Use of High- and Low-Thrust Propulsion in Combination, J. Astronaut. Sci. 10 (1963) 107-113.

[17] J. Horsewood, Interplanetary Trajectory Analysis for Combined High and Low-Thrust Propulsion Systems, Preprint. (1966).

[18] G.A. Hazelrigg, Optimal space flight with multiple propulsion systems., J. Spacecr. Rockets. (1968) 12331235.

[19] W. Dickerson, D. Smith, Trajectory optimization for solar-electric powered vehicles., J. Spacecr. Rockets. 5 (1968).

[20] L.H. Fishbach, Multiple Thrust Level Trajectories for Minimum Propellant Consumption, Case Institute of Technology, Cleveland, Ohio, 1967.

[21] E.A. Willis Jr., L.H. Fischbach, Comment on “Optimal Space Flight with Multiple Propulsion Systems,” J. Spacecr. Rockets. 6 (1969) 767. doi:10.2514/3.59617.

[22] C. Kluever, Spacecraft optimization with combined chemical-electric propulsion, J. Spacecr. Rockets. 32 (1995) 378-380.

[23] C. Kluever, Optimal Earth-moon trajectories using combined chemical-electric propulsion, J. Guid. Control. Dyn. 20 (1997) 253-258.

[24] C. Kluever, B. Pierson, Optimal earth-moon trajectories using nuclear electric propulsion, J. Guid. Control. Dyn. 20 (1997).

[25] S. Oleson, R. Myers, C. Kluever, Advanced propulsion for geostationary orbit insertion and north-south station keeping, J. Spacecr. .... 2 (1997).

[26] C.A. Kluever, Optimal Geostationary Orbit Transfers Using Onboard Chemical-Electric Propulsion, J. Spacecr. Rockets. 49 (2012) 1174-1182. doi:10.2514/1.A32213.

[27] C.A. Kluever, Designing Transfers to Geostationary Orbit Using Combined Chemical-Electric Propulsion, J. Spacecr. Rockets. 52 (2015) 1144-1151. doi:10.2514/1.A33259.

[28] D.Y. Oh, T. Randolph, S. Kimbrell, End to End Optimization of Chemical-Electric Orbit Raising Missions, J. Spacecr. Rockets. 41 (2004) 831-839. doi:10.2514/1.13096.

[29] L. Mailhe, S. Heister, Design of a hybrid chemical/electric propulsion orbital transfer vehicle, J. Spacecr. Rockets. 39 (2002) 131-139.

[30] K.A. Ehricke, Interplanetary Mission Profiles, San Diego, 1958.

[31] R. Hoelker, R. Silber, The bi-elliptical transfer between circular coplanar orbits, Alabama, 1959.

[32] A.A. Shternfel’d, Soviet Space Science, 2nd ed., Basic books Inc, New York, 1959.

[33] D. Vallado, Fundamentals of astrodynamics and applications, 2001.

[34] S. Owens, M. Macdonald, Novel Numerical Optimisation of the Hohmann Spiral Transfer, in: 64th Int. Astronaut. Congr., Beijing, China, 2013.

[35] S. Owens, M. Macdonald, Analogy to Bi-Elliptic Transfers Incorporating High- and Low-Thrust, J. Guid. Control. Dyn. 36 (2013) 890-894. doi:10.2514/1.57917.

[36] S. Owens, M. Macdonald, An Extension and Numerical Analysis of the Hohmann Spiral Transfer, in: 63rd Int. Astronaut. Congr., Naples, Italy, 2012. 
[37] S. Owens, M. Macdonald, A Novel Approach to Hybrid Propulsion Transfers, in: 62nd Int. Astronaut. Congr., Cape Town, South Africa, 2011.

[38] S. Owens, M. Macdonald, Hohmann Spiral Transfer with Inclination Change Performed by Low-Thrust System, in: S. Tanygin, R.S. Park, T.F. Starchville Jr., L.K. Newman (Eds.), Adv. Astronaut. Sci., American Astronautical Society, 2013: pp. 715-732.

[39] IADC Space Debris Mitigation Guidelines, Inter-Agency Space Debris Coordination Committee, 2007. http://www.iadc-online.org/Documents/IADC-2002-01, IADC Space Debris Guidelines, Revision 1.pdf (accessed March 18, 2016).

[40] Support to the IADC Space Debris Mitigation Guidelines, Inter-Agency Space Debris Coordination Committee, 2014. http://www.iadc-online.org/Documents/IADC-04-06 Support to IADC Guidelines rev5.5.pdf (accessed March 18, 2016).

[41] L. Sackett, L. Malchow, T. Edelbaum, Solar electric geocentric transfer with attitude constraints: analysis, 1975.

[42] C. Kluever, S. Oleson, Direct approach for computing near-optimal low-thrust earth-orbit transfers, J. Spacecr. Rockets. 97 (1998) 1783-1800.

[43] C. Kluever, Simple guidance scheme for low-thrust orbit transfers, J. Guid. Control Dyn. 21 (1998) 19-21.

[44] C.R. McInnes, Solar Sailing: Technology, Dynamics and Mission Applications, Praxis / Springer, Chichester, UK, 2004.

[45] M. Macdonald, C. McInnes, Analytical Control Laws for Planet-Centered Solar Sailing, J. Guid. Control. Dyn. 28 (2005) 1038-1048. doi:10.2514/1.11400.

[46] M. Macdonald, C. McInnes, Realistic Earth Escape Strategies for Solar Sailing, J. Guid. Control. Dyn. 28 (2005) 315-323. doi:10.2514/1.5165.

[47] M. Macdonald, C.R. McInnes, Analytic control laws for near-optimal geocentric solar sail transfers, in: D. Spencer, D. Seybold, A. Misra, R. Lisowski (Eds.), Adv. Astronaut. Sci., UNIVELT INC, 2001: pp. 23932411.

[48] M. Macdonald, C.R. McInnes, Solar Sail Capture Trajectories at Mercury, in: AIAA/AAS Astrodyn. Spec. Conf. Exhib., Monterey, USA, 2002. doi:10.2514/6.2002-4990.

[49] M. Macdonald, C.R. McInnes, A Near-Term Roadmap for Solar Sailing, in: 55th Int. Astronaut. Congr., Vancouver, Canada, 2004.

[50] M. Macdonald, G. Hughes, C. McInnes, A. Lyngvi, P. Falkner, A. Atzei, Solar Polar Orbiter: A Solar Sail Technology Reference Study, J. Spacecr. Rockets. 43 (2006) 960-972. doi:10.2514/1.16408.

[51] M. Macdonald, C. McInnes, B. Dachwald, Heliocentric Solar Sail Orbit Transfers with Locally Optimal Control Laws, J. Spacecr. Rockets. 44 (2007) 273-276. doi:10.2514/1.17297.

[52] M. Macdonald, C. McInnes, Solar sail science mission applications and advancement, Adv. Sp. Res. 48 (2011) 1702-1716. doi:10.1016/j.asr.2011.03.018.

[53] R. Broucke, P. Cefola, On the equinoctial orbit elements, Celest. Mech. (1972).

[54] M.J.H. Walker Ireland, B and Owens, J, A Set of Modified Equinoctial Orbit Elements, in: Celest. Mech. 36, D. Reidel Publishing Company, 1985: pp. 409-419.

[55] J. Betts, Optimal interplanetary orbit transfers by direct transcription, J. Astronaut. Sci. 42 (1994) 247-268.

[56] J.R. Dormand, P.J. Prince, A family of embedded Runge-Kutta formulae, J. Comput. Appl. Math. 6 (1980) 19-26. doi:10.1016/0771-050X(80)90013-3.

[57] J. Nocedal, S.J. Wright, Numerical Optimization, Springer, 2003.

[58] A.E. Petropoulos, Simple Control Laws for Low-Thrust Orbit Transfers, in: AAS/AIAA Astrodyn. Spec. Conf., Big Sky, Montana, 2003.

[59] A.N. Grubisic, S. Clark, N. Wallace, C. Collingwood, F. Guarducci, Qualification of the T6 Ion Thruster for the BepiColombo Mission to the Planet Mercury, in: 32nd Int. Electr. Propuls. Conf., Wiesbaden, Germany, 
2011. http://erps.spacegrant.org/uploads/images/images/iepc_articledownload_1988-2007/2011index/IEPC2011-234.pdf (accessed April 28, 2015).

[60] O. Duchemin, The PPS ${ }^{\circledR}$ The PPS 5000 A New-Generation 5-kW Class Hall Thruster, in: Electr. Propuls. Innov. Compet. Work., Bruxelles, Belgium, 2014. http://espaceftp.cborg.info/epic_2014/d2_s07_3_FLTH_14-0669_PPS5000_applications_perspectives.pdf.

[61] A.L. Vampola, The Space Particle Environment, (1989). http://www.dtic.mil/docs/citations/ADA207547 (accessed July 10, 2017).

[62] J.R. Wertz, D.F. Everett, J.J. Puschell, eds., Space Mission Engineering: The New SMAD, 1st ed., Microcosm Press, Hawthorne, 2011.

[63] M. Macdonald, V. Baedescu, eds., The International Handbook of Space Technology, 1st ed., Springer Praxis, Berlin Heidelberg, Germany, 2014. doi:10.1007/978-3-642-41101-4.

[64] D. Heynderickx, B. Quaghebeur, E. Speelman, E. Daly, ESA’s Space Environment Information System (SPENVIS) - A WWW interface to models of the space environment and its effects, in: 38th Aerosp. Sci. Meet. Exhib., American Institute of Aeronautics and Astronautics, Reston, Virigina, 2000. doi:10.2514/6.2000-371. 